\title{
Hybrid Nanoparticle Composites
}

\author{
Jessica Winter, ${ }^{* a b}$ Julien Nicolas ${ }^{c}$ and Gang Ruan ${ }^{\text {def }}$
}

a William G. Lowrie Department of Chemical and Biomolecular Engineering, The Ohio State University, Columbus, Ohio, USA. E-mail: winter.63@osu.edu

b Center for Emergent Materials: An NSF MRSEC, 151 W Woodruff Ave, Columbus, Ohio, USA. Tel: +1

(614) 247-7668

c Universite' Paris-Saclay, CNRS, Institut Galien Paris-Saclay, 92296 Châtenay-Malabry, France

d Department of Biomedical Engineering, College of Engineering and Applied Sciences, Nanjing

University, China

e Institute of Materials Engineering, College of Engineering and Applied Sciences, Nanjing University, China

f Jiangsu Key Laboratory of Artificial Functional Materials, Nanjing University, China

Because of their unique, size-dependent properties, nanomaterials offer tremendous promise in many fields, including microbiology and healthcare. As the last two decades have brought significant advances in the synthesis of individual nanostructures, researchers have increasingly turned their attention to the integration of those structures into composites. Such hybrid nanocomposites marry the properties of individual nanoparticles with those of other materials to generate unique structures with synergistic and emergent capabilities. Composites may combine inorganic materials with each other or with organic polymers or naturally occurring macromolecules, like proteins. The combination of different material types enables challenges that cannot be surmounted by a single material alone to be addressed.

Nowhere is this more evident than in the fields of photodynamic and photothermal therapy (PDT and PTT). The combination of nanoparticles with hydrogels (Pushpavanam et al., DOI: 10.1039/D0TB00241K), metallic photosensitizers (Gao et al., DOI: 10.1039/D0TB00097C), or imaging agents (Wang et al., DOI: 10.1039/DOTB00364F) can expand the capabilities of these materials from therapy alone to agents capable of imaging and sensing (i.e. theranostics) or combinatorial PDT/PTT and chemotherapy. Such materials offer promise for a healthcare regime in which therapeutic efficacy can be monitored in real-time and can be delivered directly to target cells. Gao et al. (DOI: 10.1039/D0TB00097C) report novel hybrid nanocomposites for combination PTT and chemotherapy. Gold nanorods for photothermal conversion were combined with a hyaluronic acid coating (i.e. THA) that served as both a stabilizing agent and a ligand to target overexpressed CD44 receptors on tumor cell surfaces. Nanocomposites were further loaded with an anticancer copper complex (i.e. CTN) with high DNA affinity, allowing chemo-PTT under laser irradiation. The resulting AuNRs-CTN@THA nanocomposites exhibited long-term photothermal conversion capacity at physiological $\mathrm{pH}$ and $\mathrm{pH} /$ temperature dual sensitive drug release, leading to synergetic antitumor efficacy and deep tumor penetration in 3D tumor spheroid models and melanoma tumor bearing mice.

Similarly, hybrid nanocomposites are advancing the field of anti-microbial and anti-fungal coatings, with improved efficacy over that observed when nanoparticles are used in isolation (Liu et al., DOI: 10.1039/D0TB00073F; Zhu et al., DOI: 10.1039/DOTB00106F). Liu et al. (DOI: 10.1039/D0TB00073F) describe an antibacterial material system based on silver nanoclusters embedded in a bacterial cellulose hydrogel. The hybrid material was prepared by synthesizing silver nanoclusters in situ in the bacterial cellulose hydrogel. An AgNO3 solution was first adsorbed onto the surface of bacterial cellulose hydrogel fibers, by interacting with abundant $-\mathrm{OH}$ groups on the fiber surface. Silver nanoclusters were then formed in situ by introducing $\mathrm{NaBH} 4$ reducing agent and dihydrolipoic acid protecting ligands. This hybrid 
material offered long-acting antibacterial efficacy thanks to its ability to release silver in a sustained manner. Furthermore, as a natural material, the bacterial cellulose hydrogel gives the hybrid material excellent biocompatibility.

Hybrid nanoparticle composite materials can also be used to tune surface properties for improved biocompatibility (Hedtrich et al., DOI: 10.1039/C9TB02480H), sensing (Zu et al., DOI: 10.1039/C9TB02953B), and catalytic properties (Tomanin et al., DOI: 10. 1039/DOTB00299B). In Hedtrich et al. (DOI: 10.1039/C9TB02480H), we learn about hybrid materials composed of iron oxide nanoparticles and MamC, a protein associated with magnetic bacteria. These materials were synthesized via coprecipitation in the presence of MamC proteins acting as passivating agents. MamC proteins fundamentally altered the nanoparticles produced, templating their growth and providing attachment points for biomodification. Here, these materials were modified with doxorubicin (DOX), a hydrophilic chemotherapeutic. The presence of DOX altered protein absorption on the composite surface, favoring fibrinogen over albumin or clusterin (i.e., themost commonly observed protein in the absence of MamC). The ability to alter the protein corona has implications for in vivo use, as proteins adsorbed from blood or plasma can impact aggregation, stability, circulation time, and ultimately biodistribution. Such materials may offer a path to increase the biocompatibility of magnetic nanoparticles in circulation.

These articles represent a cross-section of work in an emerging field. As nanoparticle synthesis continues to be refined and enters the commercial scale, the desire to combine materials to generate new properties will only expand. Current applications focus primarily on composites generated by mixing materials together in random orientations or with core/shell morphologies that are limited in scale. Thus, future challenges will likely include methods of bottom-up assembly that can precisely organize materials into large, micro- to macro-scale composites with defined properties. Such future hybrid nanoparticle composites would have great potential to transform biological and environmental fields, spanning a wide range of applications from theranostics to antimicrobial surfaces.

Dr Jessica Winter is a Professor in the William G. Lowrie Department of Chemical and Biomolecular Engineering and the Department of Biomedical Engineering and Associate Director of the Center for Emergent Materials: an NSF MRSEC at The Ohio State University. Her research interests include nanoparticles for cancer imaging, diagnostics, and drug delivery; and cell migration in the brain tumor microenvironment. She is a member of the Chemical Engineering Technical Operating Council of the American Institute of Chemical Engineers, co-founder of Core Quantum Technologies, a fellow of AAAS and AIMBE, and a senior member of the IEEE and AIChE.

Dr Julien Nicolas received his PhD degree in 2005 from the Pierre and Marie Curie University (Paris, France), where he studied nitroxide-mediated polymerization. After a postdoctoral position at the University of Warwick (Coventry, UK) on polymer-protein bioconjugates, in 2007 he obtained a permanent CNRS researcher position at Institut Galien Paris-Saclay (Châtenay-Malabry, France) and was promoted to Director of Research at CNRS in 2016. His current research activities lie in advanced macromolecular synthesis and in the design of innovative polymer-based nanomedicines, in particular polymer nanoparticles and polymer prodrug nanocarriers for anticancer therapy. He serves as an Associate Editor for Chemistry of Materials (ACS).

Dr Gang Ruan received his PhD in chemical engineering from the National University of Singapore in 2004. He worked at Emory University as a postdoctoral researcher and worked at The Ohio State University first as a postdoctoral researcher and then as a research scientist. Currently, he is a professor of biomedical engineering at Nanjing University, China. His laboratory's research thrusts include biological delivery, nano-assembly, and brain technologies. He is a co-founder of Core Quantum Technologies Inc., which 
develops nanomaterials-based products for biomedical analysis. He serves as an Assistant Dean of the College of Engineering and Applied Sciences at Nanjing University. 